Provided for non-commercial research and education use. Not for reproduction, distribution or commercial use.

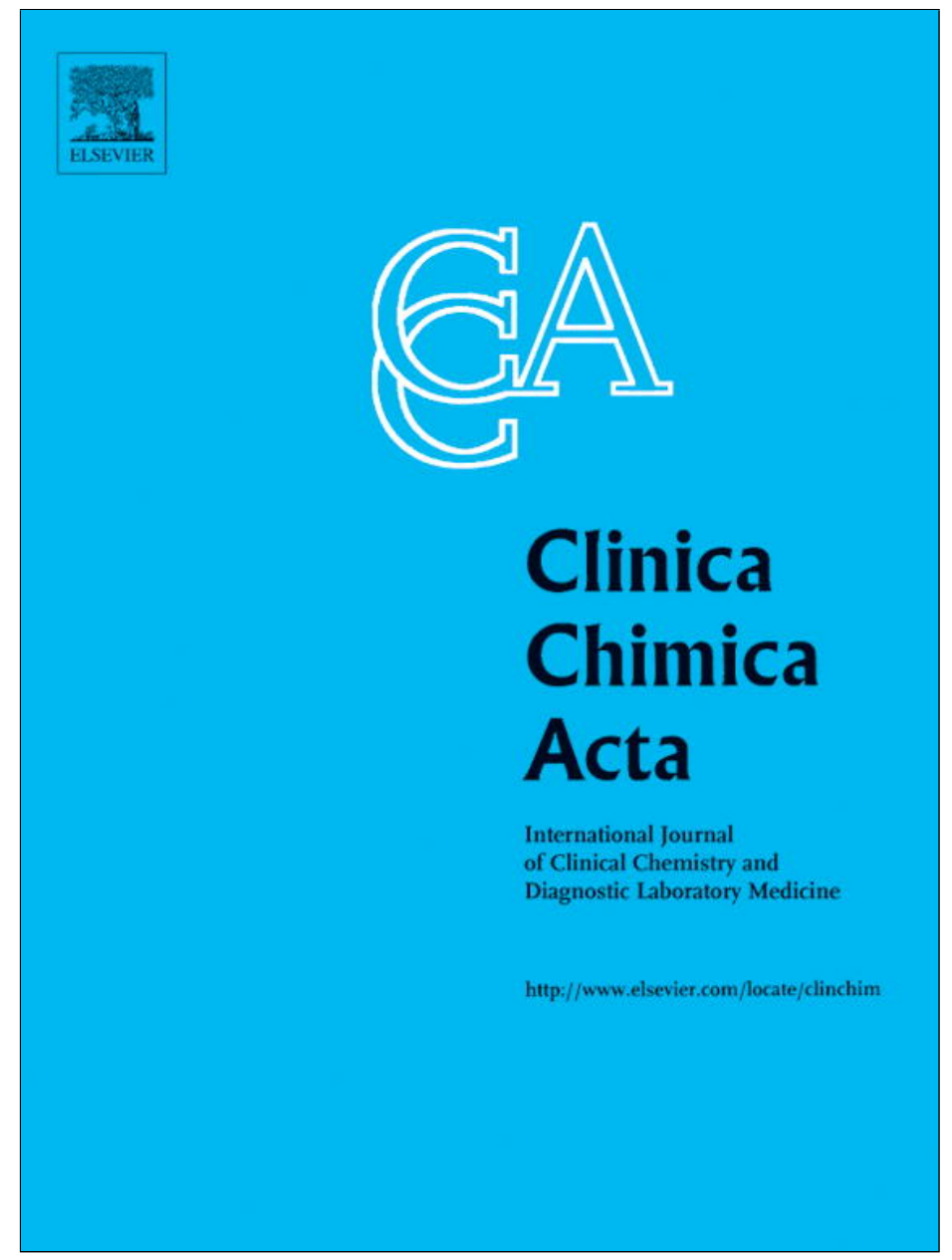

(This is a sample cover image for this issue. The actual cover is not yet available at this time.)

This article appeared in a journal published by Elsevier. The attached copy is furnished to the author for internal non-commercial research and education use, including for instruction at the authors institution and sharing with colleagues.

Other uses, including reproduction and distribution, or selling or licensing copies, or posting to personal, institutional or third party websites are prohibited.

In most cases authors are permitted to post their version of the article (e.g. in Word or Tex form) to their personal website or institutional repository. Authors requiring further information regarding Elsevier's archiving and manuscript policies are encouraged to visit:

http://www.elsevier.com/copyright 


\title{
State of the art of BNP and NT-proBNP immunoassays: The CardioOrmoCheck study
}

\author{
Aldo Clerico a,b,*, Martina Zaninotto ${ }^{\mathrm{d}}$, Concetta Prontera ${ }^{\mathrm{a}}$, Stefania Giovannini ${ }^{\mathrm{c}}$, Rudina Ndreu ${ }^{\mathrm{c}}$, \\ Maria Franzini ${ }^{\mathrm{a}, \mathrm{b}}$, Gian Carlo Zucchelli ${ }^{\mathrm{c}}$, Mario Plebani ${ }^{\mathrm{d}}$ \\ and On behalf of the Study Group on Cardiovascular Risk Biomarkers of the Italian Society of Clinical \\ Biochemistry (SIBIOC)
}

a Fondazione Toscana G. Monasterio, Pisa, Italy

b Scuola Superiore Sant'Anna, Pisa, Italy

c CNR Institute of Clinical Physiology and QualiMedLab srl, Pisa, Italy

d Department of Laboratory Medicine, University-Hospital, Padova, Italy

\section{A R T I C L E I N F O}

\section{Article history:}

Received 22 June 2012

Received in revised form 19 July 2012

Accepted 26 July 2012

Available online 15 August 2012

\section{Keywords:}

BNP

NT-proBNP

Natriuretic peptides

Immunoassay

Quality control

\begin{abstract}
A B S T R A C T
To evaluate differences in analytical performance and clinical results of BNP and NT-proBNP immunoassays, a proficiency testing program, called CardioOrmoCheck study, has been organized since 2005 under the patronage of the Study Group of the Cardiovascular Biomarkers of the Italian Society of Clinical Biochemistry (SIBIOC). On average more than 100 Italian laboratories were involved in the annual 2005-2011 cycles.

In total, 72 study samples were distributed and measured by participant laboratories for a total of 6706 results. A great difference in between-method variability was found between BNP (43.0 CV\%) and NT-proBNP (8.7 CV\%) immunoassays. However, with the only exception of the POCT method for BNP assay, all immunoassay methods showed an imprecision $\leq 10 \mathrm{CV} \%$ at the cut-off levels (i.e. $100 \mathrm{ng} / \mathrm{L}$ for BNP and $400 \mathrm{ng} / \mathrm{L}$ for NT-proBNP assay, respectively). Furthermore, CardioOrmoCheck study demonstrated that the most popular BNP immunoassays are affected by large systematic differences (on average more than 2 folds between TRIAGE Beckman-Coulter and ADVIA Centaur Siemens methods), while the agreement between NT-proBNP methods was better. CardioOrmoCheck study demonstrates that there are marked differences in analytical performance and measured values in particular among commercial methods for BNP assay. These findings suggest that it may be not reasonable to recommend identical cut-off or decision values for all BNP immunoassays.
\end{abstract}

(c) 2012 Elsevier B.V. All rights reserved.

\section{Introduction}

Cardiac natriuretic peptides, which include the atrial natriuretic peptide (ANP) and B-type natriuretic peptide (BNP) and their related peptides, constitute a complex family of peptide hormones produced and secreted by the heart [1-3]. The active peptides, ANP and BNP, are produced by cleavage of $\mathrm{COOH}$-terminal part of the pro-hormone (proANP and pro-BNP), while the N-terminal fragments of pro-hormone, NT-proANP and NT-proBNP, are currently considered inactive [1-3].

The measurement of circulating BNP and its related-peptides is now considered to be a useful marker of myocardial function [1-6], and recent international guidelines recommend its use for the diagnosis, risk stratification, and follow-up of patients with chronic or acute heart failure [5-7]. Some meta-analyses [8,9] confirmed that both BNP and NT-proBNP immunoassays have a high degree of diagnostic accuracy and clinical relevance in both acute and chronic heart failure. However

\footnotetext{
* Corresponding author at: Laboratory of Cardiovascular Endocrinology and Cell Biology, Fondazione Toascana G. Monasterio, Via Trieste 41, 56126 Pisa, Italy. Tel.: + 390585 493569; fax: + 390585493601.

E-mail address: clerico@ifc.cnr.it (A. Clerico).
}

very recent studies suggested that a great part of B-type natriuretic peptides measured in patients with cardiovascular disease is devoid of any biological activity $[2,3,10-12]$.

In order to evaluate the differences in analytical performance and clinical results of the most popular BNP and NT-proBNP immunoassays, a proficiency testing study, based on an external quality assessment scheme and called CardioOrmoCheck, have been organized and carried out in Italy since January 2005. Previous, preliminary results of the CardioOrmoCheck study suggested that there are significant differences in analytical characteristics and measured values among the most popular commercial methods for B-type related natriuretic peptides, especially among the immunoassays considered specific for BNP. In particular, a 2.7 fold difference was on average found between the BNP values measured by the two methods reporting the highest and the lowest values, respectively [13]; while the NT-proBNP immunoassays showed only slight differences in both imprecision and measured values. These differences are probably due to the different crossreactions against the precursor proBNP and other related peptides shared by BNP immunoassays [14]; although some differences in the standard material used for the curve calibration cannot be excluded. According to these data $[13,14]$, the most part of peptides measured by so-called BNP 
immunoassays in patients with heart failure may be inactive. Moreover, significant differences in imprecision among methods were previously observed in the CardioOrmoCheck study [13]. These different analytical performances, concerning both measured values and analytical imprecision, may strongly affect the clinical usefulness of BNP immunoassay methods. However, in spite of these great differences in analytical and clinical performances, the most recent international guidelines [5,7] still suggest identical decisional values for all BNP (i.e., $100 \mathrm{ng} / \mathrm{L}$ ) and NT-proBNP (i.e. $400 \mathrm{ng} / \mathrm{L}$ ) immunoassay methods.

In the present article, authors reported the results obtained by the CardioOrmoCheck study throughout all the 7 years of the activity of the collaboratirve program (i.e., from 2005 to 2011 cycle). In particular, the greatly increased number of results available (i.e., 6706 results, 3269 for BNP and 3446 for NT-proBNP assay), compared to a previous report (i.e., 2354 results) [13], allowed a better evaluation of imprecision profiles of the most popular immunoassay methods of the survey and so an accurate estimation of CV confidence intervals, concerning the decisional values. Furthermore, the increasing number of study samples with different matrix (i.e., serum, EDTA or heparin plasma), tested in the study, allowed a more accurate evaluation of the possible matrix effect on the performance of BNP and NT-proBNP immunoassays.

\section{Materials and methods}

\subsection{Collaborative study}

The CardioOrmoCheck study is a proficiency testing program for the measurement of BNP and NT-proBNP, organized in Italy since 2005 under the patronage of the Study Group of the Cardiovascular Risk Biomarkers of the Italian Society of Clinical Biochemistry (SIBIOC) by three accredited (ISO 9001 accreditation) laboratories: QualiMedLab srl, working at CNR Institute of Clinical Physiology and Fondazione Regione Toscana G. Monasterio of Pisa, Department of Laboratory Medicine, University-Hospital of Padova, and Biomedical Research Centre of Castelfranco Veneto, Italy. On average, more than 100 Italian laboratories were involved in the annual 2005-2011 cycles. In particular, in the last cycle (year 2011), 130 Italian laboratories participated in the CardioOrmoCheck study: 55 laboratories used BNP, while 75 NT-proBNP methods. The major part (about $85 \%$ ) of the participant laboratories was hospital labs, while the remaining part included private laboratories.

\subsection{Sample preparation}

In total, 72 study samples with different BNP/NT-proBNP concentrations were prepared by the central laboratories of the study (Supplemental File Table) according to the ILAC G13 guidelines, and measured by all participant laboratories for a total of 6706 determinations; some of these samples were repeatedly assayed by all laboratories to test also the within-laboratory variability. The list of BNP and NT-proBNP assay methods, more frequently used by participant laboratories, were reported in Table 1.

For the preparation of study samples, several plasma or serum specimens were pooled together to obtain a sample pool with final volume of about $100 \mathrm{~mL}$, which was immediately stored at $-20^{\circ} \mathrm{C}$. Different materials were used, such as plasma-EDTA, plasma Li-heparin, or serum (Supplemental File Table). Authors chose to prepare both plasma and serum samples in order to evaluate the relative commutability of different matrices and the possibly different degradation of peptides in plasma or serum.

All samples were tested for the absence of HBsAg, antiHCV, and antiHIV. Sample pools were prepared using residuals from samples collected from apparently healthy subjects (also divided according to gender) and patients with cardiac diseases with or without symptomatic heart failure. Blood samples collected from approximately 30-50 subjects/patients were included in every study sample. Subjects and
Table 1

List of methods more frequently used by participant laboratories for the BNP and NT-proBNP assay.

BNP assays

1. BNP assay for ARCHITECT platform Abbott Diagnostics, Abbott Park, IL, USA. In the last cycle (year 2011), $8 \%$ of the laboratories used this method. A total of 314 results were produced by all the laboratories which used this BNP method, throughout all the cycles.

2. BNP TRIAGE Biosite method for Access and UniCel DxI platforms, Beckman, Beckman Coulter, Inc., Fullerton, CA 92835, USA. In the last cycle (year 2011), $48 \%$ of the laboratories used this method. A total of 1099 results were produced by all the laboratories which used this BNP method, throughout all the cycles.

3. Triage Biosite BNP, Alere Inc., 51 Sawyer Road, Suite 200 Waltham, MA 024533448 , USA. In the last cycle (year 2011), $13 \%$ of the laboratories used this method. A total of 759 results were produced by all the laboratories which used this BNP method, throughout all the cycles.

4. Advia Centaur BNP, Siemens Healthcare Diagnostics, TarryTown, NY 10591-5097, USA. In the last cycle (year 2011), 32\% of the laboratories used this method. A total of 1016 results were produced by all the laboratories which used this BNP method, throughout all the cycles.

NT-proBNP assays

1. ECLIA NT-proBNP assay for Elecsys platform, Roche Diagnostics GmbH, D-68298 Mannheim. In the last cycle (year 2011), 32\% of the laboratories used this method. A total of 1414 results were produced by all the laboratories which used this NT-proBNP method, throughout all the cycles.

2. ECLIA NT-proBNP assay for Modular platform, Roche Diagnostics $\mathrm{GmbH}$, D-68298 Mannheim. In the last cycle (year 2011), 41\% of the laboratories used this method. A total of 1199 results were produced by all the laboratories which used this NT-proBNP method, throughout all the cycles.

3. NT-proBNP assay for Dimension platform, Siemens Medical Solutions Diagnostics, TarryTown, NY 10591-5097, USA. In the last cycle (year 2011), 6\% of the laboratories used this method. A total of 390 results were produced by all the laboratories which used this NT-proBNP method, throughout all the cycles.

4. NT-proBNP assay for Stratus system, Siemens Medical Solutions Diagnostics TarryTown, NY 10591-5097 USA. In the last cycle (year 2011), 5\% of the laboratories used this method. A total of 126 results were produced by all the laboratories which used this NT-proBNP method, throughout all the cycles.

5. NT-proBNP assay for VIDAS system, bioMérieux Italia Spa, Bagno a Ripoli, Italy. In the last cycle (year 2011), $3 \%$ of the laboratories used this method. A total of 22 results were produced by all the laboratories which used this NT-proBNP method, throughout all the cycles.

patients gave the informed consensus for the use of their residual blood samples in the study.

Study samples were sent by mail as lyophilized materials. Lyophilization procedure was performed by Polymed (Sambuca, Firenze, Italy) within two weeks after preparation of sample pools. Stored sample pools, were defrosted, then distributed in approximately 150 vials (each containing a plasma/serum volume of $0.5 \mathrm{~mL}$ ), and finally lyophilized, as previously reported [13]. The lyophilized materials were reconstituted with $0.5 \mathrm{~mL}$ of distilled water by participant laboratories before the assay. BNP and NT-proBNP concentrations of all the study samples were measured before and after the lyophilization by the reference laboratory (i.e., Laboratory of the Fondazione Regione Toscana G. Monasterio, Pisa) in order to evaluate the recovery of lyophilization procedure and the stability of BNP and NT-proBNP in the matrix samples. The recovery (mean \pm SEM) after lyophilization procedure was significantly higher ( $\mathrm{p}=0.0006$ by paired $t$ test) for NT-proBNP assay $(87.8 \pm 4.4 \%)$ than for BNP assay (66.8 $\pm 4.3 \%)$. Furthermore, the recovery was not significantly different between EDTA plasma (mean \pm SD recovery $=68.1 \pm 24.7 \%$ ), heparin plasma $(52.2 \pm 13.9 \%)$ or serum $(64.5 \%)$ samples for BNP assay [13]. Samples with unreliable results were discarded. In particular, only the samples with BNP and NT-proBNP values, which showed peptide values similar to the pathophysiological characteristics of original samples (i.e., healthy subjects, patients with moderate or more severe heart failure) [1-3], were distributed as study samples in the survey.

\subsection{Statistical analysis}

Statistical analyses of the collected results were computed by the reference laboratory (i.e., Department of Laboratory Medicine, Fondazione 
Regione Toscana G. Monasterio, Pisa), and then periodic and cumulative reports were prepared and sent by mail to each participant laboratory. It was also possible for the participant laboratories to find their individual results and the periodic and cumulative reports in a specific web site using a personal password (http://eqas.ifc.cnr.it). The periodic reports include a scoring system, reporting the performance of each single laboratory in comparison with those of other laboratories. Moreover, each report contains some quality parameters computed as follows:

1. Laboratory bias: mean percent deviations from the consensus mean of the results the laboratory obtained for all the study samples assayed in test period.

2. Laboratory imprecision: mean imprecision estimated from results reported by the laboratory for unidentified replicate samples. The mean imprecision is obtained by pooling the CVs for different replicate pools.

3. $\mathrm{CV}_{\mathrm{T}} \%$ (average between-laboratory agreement): pooled betweenlaboratory CV for all the study samples mailed out in the considered period.

4. BIAS\% (average bias): the root mean square of all the laboratory biases.

5. $\mathrm{CV}_{\mathrm{L}}$ \% (average imprecision): median of all the laboratory imprecisions, accounting for the dispersion of the results of the laboratories with respect to their own means.

6. Method bias: mean of the percent deviations from the consensus mean of all the results reported by the users of the method.

7. Method imprecision: pooled CV computed from all results reported by the users of the method.

In particular, total variability $\left(\mathrm{CV}_{\mathrm{T}} \%\right)$ was estimated by averaging the CVs computed from the results of each study sample. This variability includes both systematic between-method differences and differences introduced by the laboratories. The imprecision of the methods was estimated by averaging the CVs of the results produced by the participants (using the same method) for the same study sample. Therefore, the reported average CVs, used for the calculation of imprecision profiles, were an estimate of the within-method, betweenlaboratory imprecision achieved by the method during the multicentre collaborative study. Outlier values were estimated according to the procedure recommended by Healy [15].

\section{Results}

\subsection{Assay methods}

The most important observation regarding the CardioOrmoCheck study is the inversion in the utilization trend of B-type natriuretic peptide immunoassays observed in the last years of the study. Indeed, while in the previous report [13] BNP assay methods were more utilized than NT-proBNP ones (53\% vs 43\%), at present time, NT-proBNP immunoassays resulted more utilized by Italian laboratories with 3446 results (51\%) compared to 3260 (49\%) results of BNP immunoassays. In particular, the ECLIA system for NT-proBNP, using the Elecsys and Modular platforms, was the most utilized method with a mean utilization ratio of $39 \%$ (on average $76 \%$ of utilization ratio considering only the NT-proBNP methods).

The MEIA method for the AxSYM system (Abbott Diagnostics) was gradually replaced in the last 3 years of the study cycles by the chemiluminescent assay using the ARCHITECT platform (Abbott Diagnostics). Some methods, including Immulite 2500, Vista and Stratus systems for NT-proBNP assay, were scarcely utilized by the participant laboratories, and so only a low number of results were available (Table 1). For this reason, the results of these methods were not considered in some statistical analyses of the present study.

\subsection{Variability estimation}

The mean total variability (i.e., including variability among all methods and laboratories) for BNP methods (43.0 CV\%) was greatly higher than that for NT-proBNP methods (8.7 CV\%). For BNP immunoassays, the mean variability, due to the difference between-methods (39.9 $\mathrm{CV} \%$ ), included the predominant part of total variability (corresponding to $86 \%$ of total variability), being the within-method variability on average $16.0 \mathrm{CV} \%$. On the contrary, for NT-proBNP immunoassays the within-method and between-method variabilities were $6.5 \mathrm{CV} \%$ and $5.8 \mathrm{CV} \%$, respectively; thus suggesting that the between-method variability contributes to the total variability less than within-method variability ( $44 \%$ vs $56 \%$, respectively).

In order to better characterize the differences in imprecision between BNP and NT-proBNP immunoassays, we evaluated the imprecision profiles of the immunoassays methods, which were more popular in the CardioOrmoCheck study and presented an adequate number of data for the statistical analysis (more than 350 results) (Table 2). In particular, CV data of the MEIA method using AxSYM system and those of the chemiluminescent assay ARCHITECT platform for BNP assay, as well as the STRATUS, VISTA and VIDAS systems for NT-proBNP were not analyzed owing to an inadequate number of available data. For the other immunoassays (Table 1), the relationships (bivariate plots) between the imprecisions, expressed as CV \% (Y-axis), and measured concentrations of the peptide (X-axis) were reported in Figs. 1 and 2 for BNP and NT-proBNP immunoassays, respectively.

As far as the BNP methods are concerned, a great variability in the imprecision was found between BNP immunoassays ( $p<0.0001$ by repeated measures ANOVA). In particular, the TRIAGE POCT method for BNP showed significantly higher imprecision than the ADVIA Siemens and TRIAGE Beckman-Coulter automated systems $(\mathrm{p}<0.0001$ by Scheffè post hoc test after ANOVA). Furthermore, we divided the 72 study samples in 3 groups according to the BNP concentrations: group $\mathrm{A}, \mathrm{BNP} \leq 50 \mathrm{ng} / \mathrm{L}$; group B, BNP ranging from 51 to $100 \mathrm{ng} / \mathrm{L}$; group C, $\mathrm{BNP}>100 \mathrm{ng} / \mathrm{L}$. The variation of CV values (dependent variable) was significantly explained ( $<<0.0001$ by two way ANOVA) by differences in both BNP values, as measured by different immunoassays, and group concentrations. These data indicate that the imprecision of BNP immunoassays is strongly dependent by peptide concentrations with higher imprecision at lower values (Fig. 1). As a result, assuming a linear relationship between $\mathrm{CV}$ and logarithmic transformed values of the measured concentration, it was possible to estimate for the most common immunoassays in the survey the imprecision at the cut-off value of $100 \mathrm{ng} / \mathrm{L}$, which is the decisional value recommended by international guidelines [5,7]; these data are reported in Table 2.

As far as the imprecisions of NT-proBNP immunoassays (Fig. 2) are concerned, a lower variability of imprecision was found compared to that of BNP immunoassays (Fig. 1). We divided the study samples in 3

Table 2

Imprecision data on the most used BNP and NT-proBNP immunoassays in the study.

\begin{tabular}{lcc}
\hline System & Results & $\begin{array}{l}\text { CV }(\mathrm{CI} 95 \%)^{\mathrm{a}} \\
(\%)\end{array}$ \\
\hline BNP immunoassays & & \\
ADVIA Siemens & 1016 & $10.2(0.4-20)$ \\
POCT TRIAGE Alere & 759 & $19.6(2.3-36.9)$ \\
TRIAGE Beckman-Coulter & 1099 & $9.5(6-2-12.8)$ \\
NT-proBNP immunoassays & & $5.7(5.3-6.2)$ \\
ECLIA Modular Roche & 1199 & $5.7(5.2-6.2)$ \\
ECLIA Elecsys Roche & 1414 & $9.9(8.9-10.9)$ \\
Dimension Siemens & 390 & \\
\hline
\end{tabular}

a For BNP immunoassays, the CV values and the $95 \%$ respective confidence intervals (CI) were calculated at the cut-off (decisional) value (i.e. $100 \mathrm{ng} / \mathrm{L}$ ) [5,7] by assuming a linear relationship between CV (dependent variable) and peptide concentration (independent variable) values. For NT-proBNP immunoassays, the imprecision values reported in the table represent the mean $\mathrm{CV}$ values and the respective confidence intervals, calculated by pooling together all control samples. 
A)

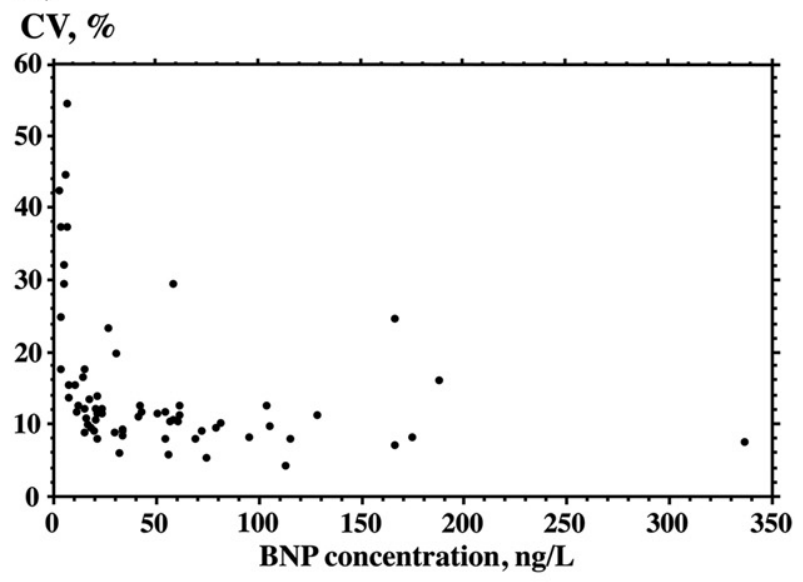

B)

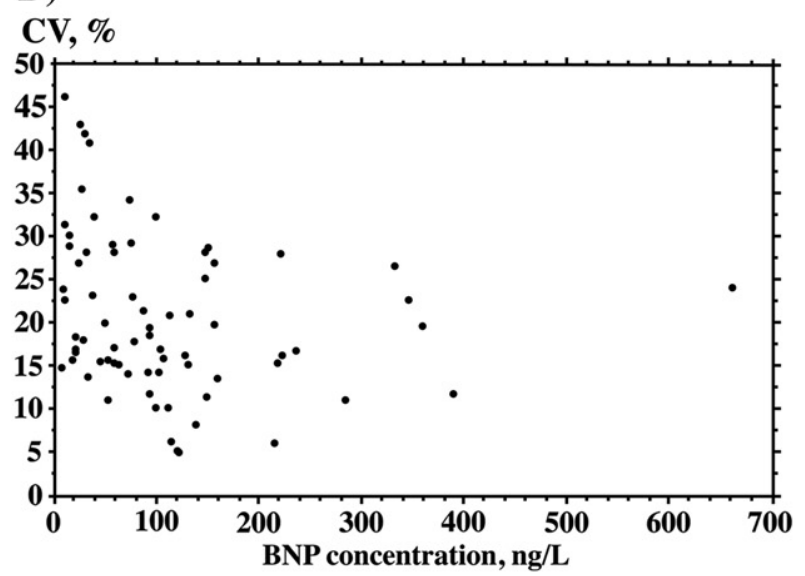

C)

\section{$\mathrm{CV}, \%$}

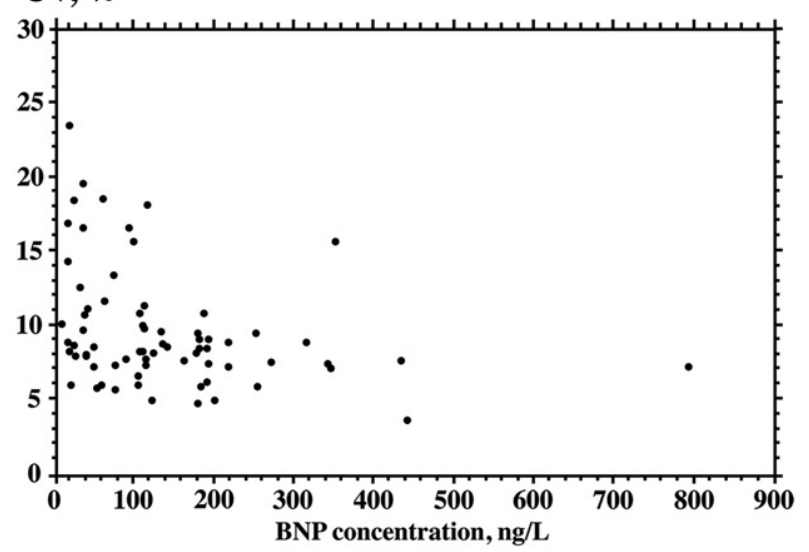

Fig. 1. Bivariate plots of relationship between $\mathrm{CV}$ values (Y-axis) and BNP concentrations (X-axis) measured by the most popular immunoassays in the survey. Part A. Relationship between CV values and BNP concentrations measured by ADVIA Siemens automated systems. Part B. Relationship between CV values and BNP concentrations measured by TRIAGE POCT Alere method. Part C. Relationship between CV values and BNP concentrations measured by TRIAGE Beckman-Coulter automated systems.

groups according to the peptide concentrations: group A, NTproBNP $\leq 800 \mathrm{ng} / \mathrm{L}$; group $\mathrm{B}$, NT-proBNP ranging from 801 to 2000 ng/l; group C, NT-proBNP values $>2000 \mathrm{ng} / \mathrm{L}$. We performed a two way, repeated measures, ANOVA using CV values as dependent variable and concentration groups and immunoassay methods as two independent variables. The mean imprecision of the automated
A)

$\mathrm{CV}, \%$

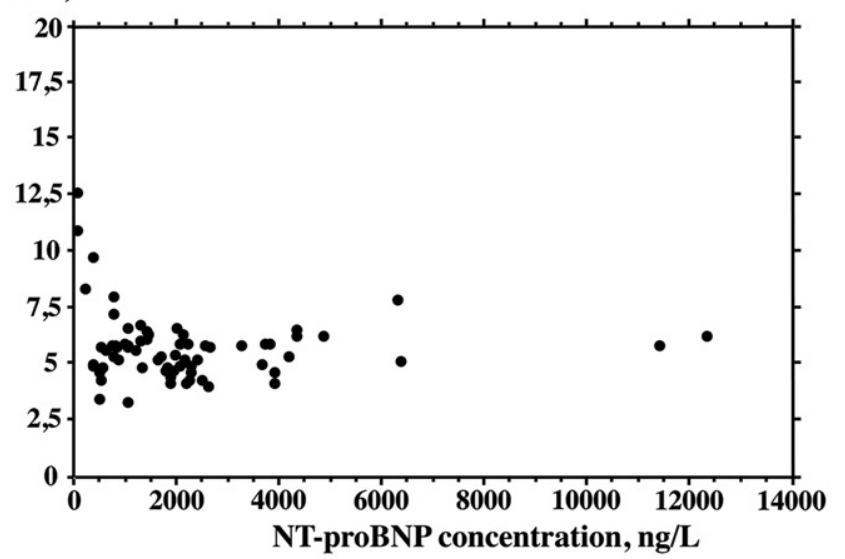

B)

$\mathrm{CV}, \%$

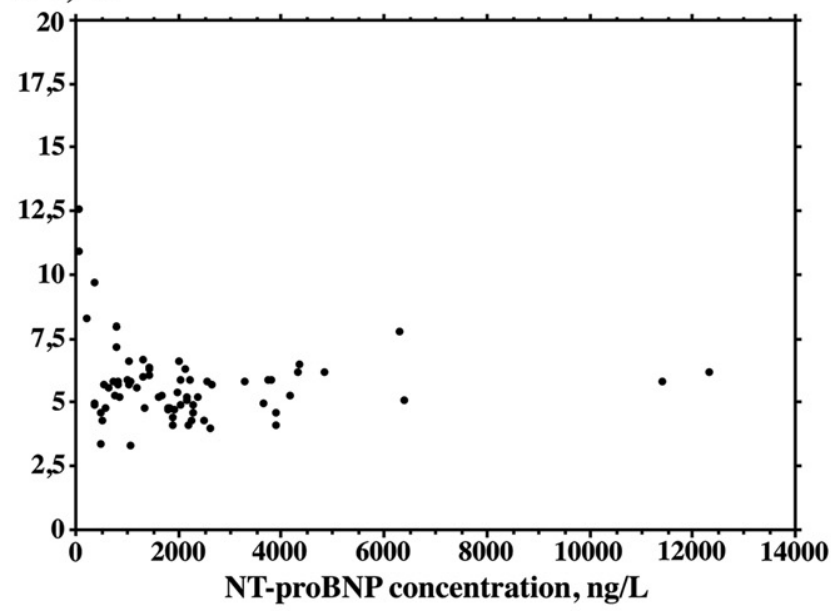

C)

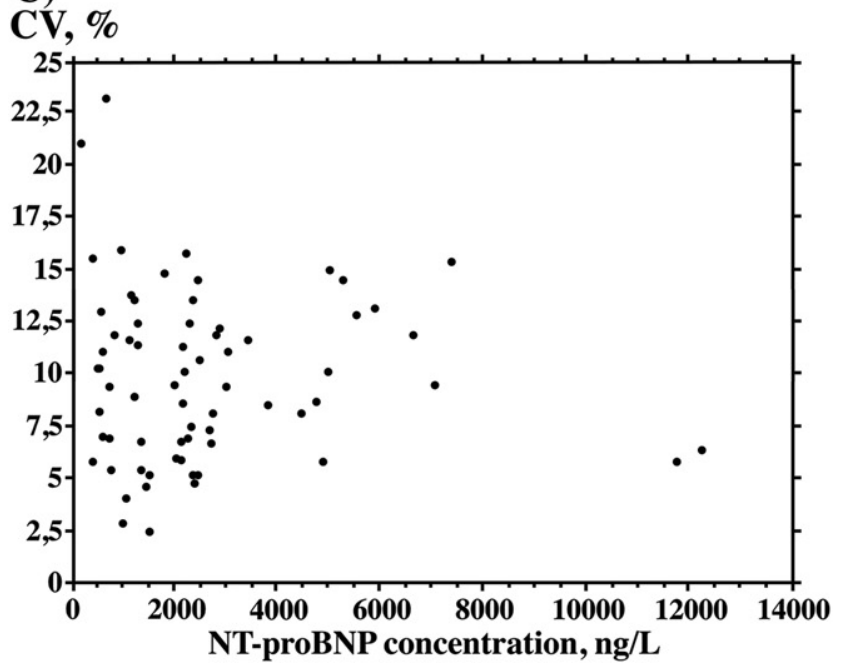

Fig. 2. Bivariate plots of relationship between $\mathrm{CV}$ values and NT-proBNP concentrations measured by the most popular immunoassays in the survey. Part A. Relationship between CV values and NT-proBNP concentrations measured by ECLIA method by Elecsys platform (Roche Diagnostics). Part B. Relationship between CV values and NT-proBNP concentrations measured by ECLIA method by Modular platform (Roche Diagnostics). Part C. Relationship between CV values and NT-proBNP concentrations measured by Dimension platform (Siemens Medical Solutions Diagnostics). 
platform dimension was found to be significantly higher $(\mathrm{p}<0.0001$ by Scheffé post hoc test) than those of ECLIA methods using both Elecsys and Modular platforms (Table 2). We found no association $(\mathrm{p}=0.4623)$ between the concentration groups (independent variable) and CV (dependent variable) by two ways, repeated measures ANOVA. Moreover, there were no significant correlations between CV values and log-transformed NT-proBNP concentrations for all the immunoassays. As a result, assuming that the imprecision at the cut-off level (i.e. $400 \mathrm{ng} / \mathrm{L}$ ) is not significantly different to the mean imprecision, we estimated the $95 \%$ confidence intervals of the mean imprecision for each NT-proBNP immunoassay by pooling all data together (Table 2).

\subsection{Evaluation of bias}

As far as the BNP immunoassays are concerned, these methods gave results closely related with correlation coefficient values ranging from $\mathrm{R}=0.920$ (correlation between the TRIAGE POCT method and ADVIA Siemens) to 0.981 (correlation between the TRIAGE Beckman-Coulter system and the TRIAGE POCT method) (Table 3). However, a significant difference $(\mathrm{p}<0.0001$ by Scheffé post hoc test after repeated measures ANOVA using log-transformed data) was found between the mean BNP values measured by immunoassay methods (Fig. 3A). In particular, on average ADVIA systems (mean $\pm S D=50.6 \pm 56.9 \mathrm{ng} / \mathrm{L}$ ) showed values about half $(\mathrm{p}<0.0001$ by Scheffé test after ANOVA using logtransformed data) than those of TRIAGE POCT method (110.6 \pm $109.8 \mathrm{ng} / \mathrm{L})$, TRIAGE Beckman-Coulter systems (139.6 $\pm 127.2 \mathrm{ng} / \mathrm{L}$ ), and MEIA Abbott systems $(93.4 \pm 100.1 \mathrm{ng} / \mathrm{L})$, respectively. As an example, regression line and Bland-Altman plot concerning the BNP results obtained with ADVIA Centaur and TRIAGE Beckman-Coulter systems are reported in Fig. 4A and B, respectively. A significant bias, which increases linearly with the increase in BNP concentration, was obtained (Fig. 4B). Finally, the matrix effect on the results of BNP immunoassays was also tested by means of two way, repeated measures ANOVA. Plasma EDTA samples showed a significantly different $(p=0.0066)$ behavior compared to the other matrices (i.e., serum and heparin samples). These findings indicate that EDTA plasma samples are preferable for a proficiency testing study, such as the CardioOrmoCheck.

As far as the NT-proBNP immunoassays are concerned, these methods gave results closely related: the two ECLIA methods showed a correlation $\mathrm{R}$ value corresponding to 0.948 , while the Dimension methods showed $\mathrm{R}$ values corresponding to 0.938 with ECLIA Elecsys and to 0.982 with ECLIA Modular, respectively (Table 3). Finally, a significant difference $(\mathrm{p}<0.0001$ by Scheffé post hoc test after repeated measures ANOVA using log-transformed data) was also found between the mean NT-proBNP values measured with the two ECLIA methods and Dimension platform system, although smaller than that seen for the BNP methods (Fig. 3B). Indeed, Dimension method showed NTproBNP values on average about $17 \%$ higher than the two ECLIA methods. Finally, the different matrices tested in the study (serum, EDTA or heparin

Table 3

Correlation matrix between the values measured with the most popular BNP/NT-proBNP methods in study samples.

\begin{tabular}{|c|c|c|c|c|}
\hline \multicolumn{5}{|c|}{ Part A. BNP immunoassay methods } \\
\hline Methods & ADVIA & POCT & BNP TRIAGE & MEIA \\
\hline ADVIA & 1.000 & 0.920 & 0.941 & 0.975 \\
\hline POCT & 0.920 & 1.000 & 0.981 & 0.930 \\
\hline BNP TRIAGE & 0.941 & 0.981 & 1.000 & 0.945 \\
\hline MEIA & 0.975 & 0.930 & 0.945 & 1.000 \\
\hline
\end{tabular}

Part B. NT-proBNP immunoassay methods

\begin{tabular}{llll}
\hline Methods & Elecsys & Modular & Dimension \\
\hline Elecsys & 1.000 & 0.948 & 0.938 \\
Modular & 0.948 & 1.000 & 0.982 \\
Dimension & 0.938 & 0.982 & 1.000 \\
\hline
\end{tabular}

A)

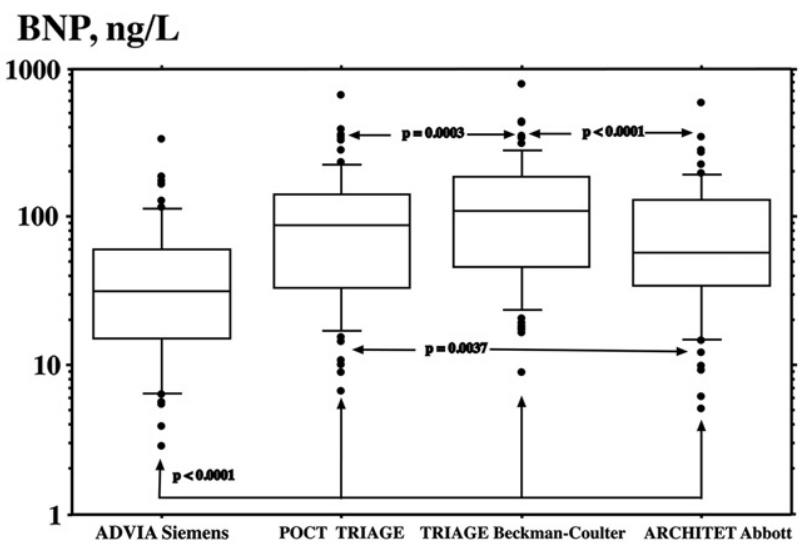

B)

NT-proBNP, ng/L

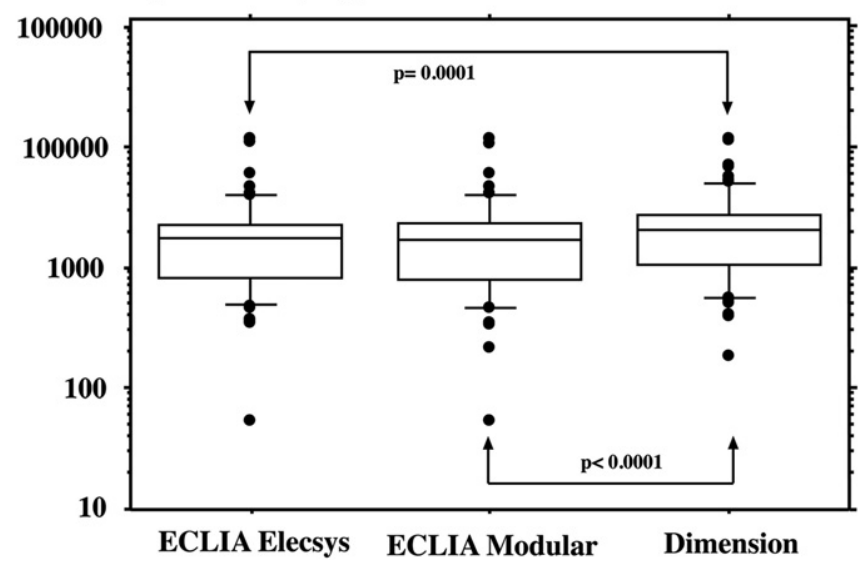

Fig. 3. Box (distribution) plot of BNP (Part A) and NT-proBNP (Part B) values measured by the most popular methods of the study. The data are reported as boxes indicating the 10th, 25th, 50th (median), 75th and 90th percentiles of BNP and NT-proBNP values measured in the 72 study samples; the outliners were indicated as separated black circles. The concentrations (Y-axis) are reported as log-scale. The levels of statistical significance ( $\mathrm{p}$ values) are also indicated in the figure.

samples) did not significantly $(\mathrm{p}=0.7688)$ affect the results of NT-proBNP immunoassays, as tested by means of two way, repeated measures ANOVA.

\section{Discussion}

\subsection{Study protocol}

Several issues should be considered when some immunoassay methods for the measurement of B-type related natriuretic peptides should be evaluated in a proficiency testing study [13]. In particular, some critical points are the low stability in vitro of B-type biologically active peptides, the possible matrix effects, and the evaluation of commutability of study samples. Considering these critical issues, to perform a reliable proficiency testing study for BNP and NT-proBNP immunoassay a formidable task should be considered.

Taking into account quality specifications [16] and recommendations by manufacturers [17], several different matrices derived from human blood samples should be used in a proficiency testing study [13] for BNP and NT-proBNP immunoassays. Indeed, the quality specifications for BNP assay recommend the use of EDTA plasma samples [16], while serum should be preferred for NT-proBNP assay [16,17]. Accordingly, one of the most important aims of the CardioOrmoCheck study was the evaluation of relative commutability of different matrices. 
A)

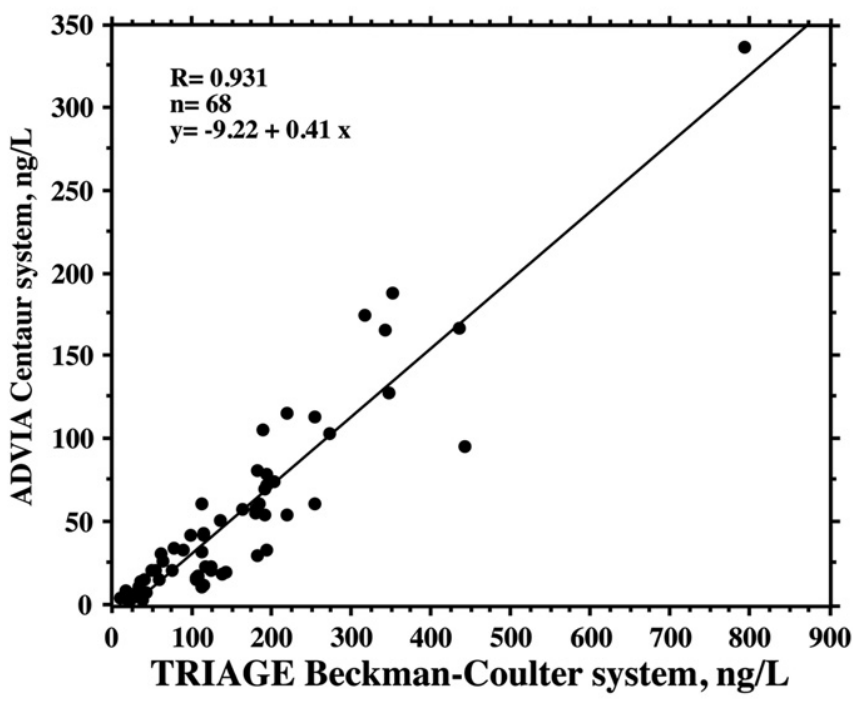

B)

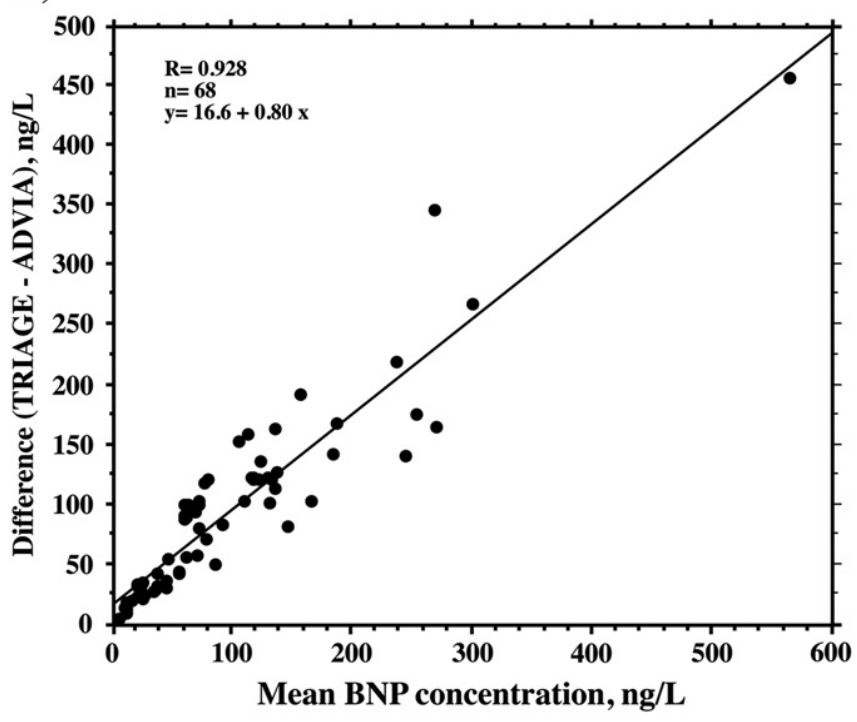

Fig. 4. Part A. Linear regression between the BNP values measured with TRIAGE Beckman-Coulter (X-axis) and ADVIA Centaur (Y-axis) systems in 68 study samples. Part B. Linear regression (Bland-Altman plot) between the mean BNP concentration, measured with TRIAGE Beckman-Coulter and ADVIA Centaur systems (X-axis), and the difference (Y-axis) between the BNP values measured by these two methods (TRIAGE - ADVIA) in 68 study samples.

At least to our knowledge, a primary reference material, which is certified as commutable for all BNP and NT-proBNP immunoassays, is not available. As a result, it is not possible to prepare secondary reference materials, calibrators or control samples with a certified concentration of the peptide [18-20]. It seems to be not reasonable to use in the present study a procedure recommended for the preparation of primary reference materials, such as the CLSI C53-A protocol [21]. Indeed, the aim of the present study is only to evaluate the intra- and between-assay variability of BNP and NT-proBNP immunoassays, which is an objective different to the more ambitious intendment to calibrate or to assess the trueness of these methods. According to this aim, as also suggested by other Authors [19,20], if commutable reference materials suitable for direct use are lacking, the only possible alternative for establishing traceability is to split human fresh samples with a laboratory performing the reference measurement procedure. However, the reference procedure for the measurement of B-type related peptides is also lacking. In a proficiency study, when the reference material and method are both lacking, the only remaining and practicable way is to compare the results of the different methods with the consensus mean, as made in CardioOrmoCheck study. Evidently, the "consensus mean" approach can allow only a relative estimate of bias between methods and laboratories. In addition, it is possible to test only the relative commutability of study samples, distributed in the proficiency testing program, in comparison with the original samples collected from healthy subjects or patients (but not with a reference material).

It is well known that some B-type related peptides, and especially the active peptide, $\mathrm{BNP}_{1-32}$, are greatly degradable both in vivo and in vitro [2,3]. Preliminary data performed in the reference laboratory of the CardioOrmoCheck study (data not shown) indicated that it is not possible to organize a reliable proficiency study for BNP immunoassays, including more than 100 laboratories, using frozen plasma specimens, as study samples. As a result, in order to reduce the in vitro degradation and to make the distribution as well as the measurement of blood samples easier, only lyophilized plasma and serum samples were used in the CardioOrmoCheck study. As previously reported [13], the recovery after lyophilization procedure was significantly higher for NT-proBNP assay (on average 88\%) than for BNP assay (on average 67\%). The lower recovery after lyophilization and the greater instability in vitro of the BNP compared to NT-proBNP, may be, almost in part, the causes of greater variability found between BNP and NT-proBNP immunoassays in the present study. However, other authors [22-25] also reported a great variability in the ratio of BNP and NT-proBNP among both individual subjects and patients with cardiac diseases.

The term commutability has been defined by the CLSI C53-A protocol "as the interassay properties of reference material, calibrator, or trueness quality control materials that are comparable with those demonstrated by authentic clinical specimens" [21]. In the present study, a progressive increase in both BNP and NT-proBNP values of the consensus means from healthy subjects to patients with severe heart failure was found (Supplemental File Table), thus suggesting that the study samples, used in the CardioOrmoCheck study, retained (almost in part) the clinical information of the original blood samples. Furthermore, the good agreement between BNP or NT-proBNP methods, as expressed by the correlation coefficients (Table 3), found for the study samples, was very similar to those previously reported by several head-to-head comparisons of analytical characteristics and clinical results of immunoassay methods performed by the reference laboratory of the CardioOrmoCheck study [26-34] or other laboratories [22-25,35-37], using authentic specimens collected from healthy subjects and patients with cardiac or extracardiac diseases.

According to the data discussed in the previous paragraphs, the results of the present study confirm our preliminary data [13], suggesting that it is possible to prepare suitable study samples for a proficiency testing program by pooling human blood specimens collected from healthy subjects or patients with cardiac disease.

\subsection{Results discussion}

The CardioOrmoCheck study, including results concerning 7 years (from 2005 to 2011), indicates that the panorama of commercial BNP and NT-proBNP methods is rapidly changing. The immunoradiometric assay method for BNP assay by Shionogi, evaluated in the previous study [13], is at present no more commercialized, at least in Europe. On the other hand, the BNP method for ARCHITECT platform by Abbott, as well as VISTA, STRATUS, and IMMULITE systems by Siemens, and VIDAS system by BioMérieux for proBNP assay, not evaluated in the previous study [13], have been only recently distributed and so these "new" methods have been adopted by participating laboratories only in the last 2 or 3 cycles of the CardioOrmoCheck study. Unfortunately, the results concerning these "new" BNP and NT-proBNP methods are insufficient to allow a reliable statistical analysis.

Since BNP and NT-proBNP share completely different biochemical structure, molecular weight, biological activity and degradation pathways 
[1-3,10-12], it is not surprising that BNP and NT-proBNP immunoassays show not only different analytical characteristics and quality specifications, but also different clinical results. Indeed, data of the CardioOrmoCheck study confirm that there are wide differences in both analytical performances and measured peptide values, especially among BNP methods. These differences in the performance between BNP and NT-proBNP immunoassays are theoretically expected because all the commercial NT-proBNP methods tested in the study (the two ECLIA methods and Dimension method) actually use antibodies and standard materials from the same source (i.e., Roche Diagnostics), while BNP methods use different antibodies and standard materials $[14,16,22]$.

The greater number of results obtained in the present study allowed a more complete analysis of analytical performance of immunoassay methods than the previous one [13]. In particular, it was possible to evaluate the imprecision profiles and to calculate the imprecision at the recommended cut-off values for the most popular BNP and NT-proBNP immunoassays of the survey. The results of CardioOrmoCheck study demonstrate that the imprecision around the recommended cut-off values (corresponding to $100 \mathrm{ng} / \mathrm{L}$ for BNP and $400 \mathrm{ng} / \mathrm{L}$ for NT-proBNP, respectively) [5,7] varies among methods (Table 2). However, our data indicate that with the only exception of POCT method for BNP assay, all other BNP and NT-proBNP immunoassays show an imprecision at the cut-off values lower or around the $10 \% \mathrm{CV}$. On average, the NT-proBNP methods showed a lower variability compared to BNP immunoassays. These data are well in agreement with those previously reported in a study performed in the reference laboratory of the CardioOrmoCheck study, which compared the analytical characteristics of some BNP immunoassays with those of the ECLIA method for NT-proBNP [27]. This difference is probably in great part due to the different biochemical characteristics of BNP and NT-proBNP. NT-proBNP shows greater molecular mass and higher circulating levels, and it is more stable in vivo and in vitro than BNP. As a result, it is easier to set up a robust immunoassay method for NT-proBNP than for BNP.

As far as the bias between the BNP immunoassays is concerned, the results of the CardioOrmoCheck study confirm that the most popular BNP immunoassays are affected by large systematic differences (on average more than 2 fold between TRIAGE Beckman-Coulter and ADVIA Centaur Siemens methods, Figs. 3A and 4). Wide differences between the results of BNP immunoassays were also previously reported in a study performed in the reference laboratory of the CardioOrmoCheck study [27]. In particular, this study found lower BNP values measured by ADVIA Centaur and IRMA by Shionogi methods compared to the other immunoassays, such as the POCT TRIAGE methods and the MEIA methods for the AXSYM platform [27].

As far as the NT-proBNP immunoassays are concerned, Di Serio et al. [35] reported that some EDTA plasma samples (5 samples included in a set of 65) showed unexplainable higher NT-proBNP concentrations with Dimension platform compared to heparin plasma samples. As a result, these authors [35] suggested the use of heparin rather than EDTA plasma samples for the measurement of NT-proBNP. Prontera et al. [28] observed slightly but significant lower NT-proBNP values for EDTA plasma samples than for serum or heparin plasma samples with the ECLIA method. As a result, the significant bias found in the present study between the NT-proBNP values measured by the two ECLIA methods and the Dimension system in the study samples may be (almost in part) due to matrix effects. However, our data indicate that the different matrices of study samples (serum, EDTA or heparin samples) do not significantly affect the results of NT-proBNP immunoassays. It is important to note that Roche Diagnostics recently introduced a new ECLIA method for the NT-proBNP assay, which uses monoclonal antibodies instead of polyclonal antibodies of the previous method [28]. A recent study performed in the reference laboratory of the CardioOrmoCheck study [28] reported that the monoclonal ECLIA method showed very similar analytical characteristics with slightly lower NT-proBNP results (on average $-2.5 \%$ ) than the polyclonal ECLIA method. However, the data collected so far in the CardioOrmoCheck study are not sufficient to demonstrate a significant difference between the results of polyclonal compared to monoclonal ECLIA method.

\subsection{Prospective and conclusive remarks}

The data of the CardioOrmoCheck study indicate that the panorama of commercial BNP and NT-proBNP methods is rapidly changing. The most important observation regarding the CardioOrmoCheck study is the inversion in the utilization trend of B-type natriuretic peptide immunoassays observed in the last years of the study, indicating that in these years the NT-proBNP immunoassays resulted more utilized by Italian laboratories than the BNP immunoassays. As suggested by some authors [38,39], it is also conceivable that some methods, more specific for the intact prohormone peptide (proBNP), may soon become commercially available $[40,41]$. These data clearly suggest that there is not yet available an ideal method for the measurement of B-type natriuretic peptides. The proficiency testing study, such as the CardioOrmoCheck study, may support the comparative evaluation with the aim to find the most reliable methods and to achieve a better harmonization between immunoassay methods for BNP assay.

Moreover, the results of the CardioOrmoCheck study demonstrate that there are marked differences in analytical performance and measured values especially among commercial methods for BNP assay. These findings suggest that it may be not reasonable to suggest identical cut-off or decision values for all BNP immunoassays, as recommended by international guidelines [5,7]. Further studies are needed to confirm this observation.

Supplementary data to this article can be found online at http:// dx.doi.org/10.1016/j.cca.2012.07.017.

\section{References}

[1] Clerico A. Pathophysiological and clinical relevance of circulating levels of cardiac natriuretic hormones: are they merely markers of cardiac disease? Clin Chem Lab Med 2002;40:752-60.

[2] Clerico A, Giannoni A, Vittorini S, Passino C. Thirty years of the heart as an endocrine organ: physiological role and clinical utility of cardiac natriuretic hormones. Am J Physiol Heart Circ Physiol 2011;301:H12-20.

[3] Clerico A, Vittorini S, Passino C. Measurement of the pro-hormone of brain type natriuretic peptide (proBNP): methodological considerations and pathophysiological relevance. Clin Chem Lab Med 2011:4:1949-54.

[4] Tang WH, Francis GS, Morrow DA, et al. National Academy of Clinical Biochemistry Laboratory Medicine practice guidelines: clinical utilization of cardiac biomarker testing in heart failure. Circulation 2007;116:e99-109.

[5] Dickestein K, Cohen-Solal A, Filippatos G, et al. The Task Force for the Diagnosis and Treatment of Acute and Chronic Heart Failure of the European Society of Cardiology. ESC guidelines for the diagnosis and treatment of acute and chronic heart failure 2008. Eur J Heart Fail 2008;10:933-89.

[6] Jessup M, Abraham WT, Casey DE, et al. 2009 focused update: ACCF/AHA guidelines for the diagnosis and management of heart failure in adults: a report of the American College of Cardiology Foundation/American Heart Association task force on practice guidelines: developed in collaboration with the International Society for Heart and Lung Transplantation. Circulation 2009;119:1977-2016.

[7] NICE, cinical guieline no 108. Chronic heart failure. National clinical guideline for diagnosis and management in primary and secondary care; August 2010. p. 1-222.

[8] Clerico A, Fontana M, Zyw L, Passino C, Emdin M. Comparison of the diagnostic accuracy of brain natriuretic peptide (BNP) and the N-terminal part of the propeptide of BNP immunoassays in chronic and acute heart failure: a systematic review. Clin Chem 2007;53:813-22.

[9] Ewald B, Ewald D, Thakkinstian A, Attia J. Meta-analysis of B type natriuretic peptide and $\mathrm{N}$-terminal pro B natriuretic peptide in the diagnosis of clinical heart failure and population screening for left ventricular systolic dysfunction. Intern Med J 2008;38: 101-13.

[10] Liang F, O'Rear J, Schellenberger U, et al. Evidence for functional heterogeneity of circulating B-type natriuretic peptide. J Am Coll Cardiol 2007;49:1071-8.

[11] Seferian KR, Tamm NN, Semenov AG, et al. The brain natriuretic peptide (BNP) precursor is the major immunoreactive form of BNP in patients with heart failure. Clin Chem 2007;53:866-73.

[12] Hammerer-Lercher A, Halfinger B, Sarg B, et al. Analysis of circulating forms of proBNP and NT-proBNP in patients with severe heart failure. Clin Chem 2008;54: 858-65

[13] Prontera C, Zaninotto M, Giovannini S, et al. Proficiency testing project for brain natriuretic peptide (BNP) and the N-terminal part of the propeptide of BNP 
(NT-proBNP) immunoassays: the CardioOrmoCheck study. Clin Chem Lab Med 2009; $47: 762-8$.

[14] Luckenbill KN, Christenson RH, Jaffe AS, et al. Cross-reactivity of BNP, NT-proBNP, and proBNP in commercial BNP and NT-proBNP assays: preliminary observations from the IFCC Committee for Standardization of Markers of Cardiac Damage. Clin Chem 2008;54:619-21.

[15] Healy MJ. Outliers in clinical chemistry quality-control schemes. Clin Chem 1979;25:675-7.

[16] Apple FS, Panteghini M, Ravkilde J, et al. On behalf of the Committee on Standardization of Markers of Cardiac Damage of the IFCC. Quality specifications for B-type natriuretic peptide assays. Clin Chem 2005;51:486-93.

[17] Siemens Technical Bulletin. Dimension clinical chemistry system: heterogeneous immunoassay module; 2008. REF.RF423A.

[18] Clinical and Laboratory Standards Institute. Metrological traceability and its implementation; a report. CLSI document X5-R. Wayne, PA: CLSI; 2006.

[19] Panteghini M. Traceability as a unique tool to improve standardization in laboratory medicine. Clin Biochem 2009;42:236-40.

[20] Miller WG. Specimen materials, target values and commutability for external quality assessment (proficiency testing) schemes. Clin Chim Acta 2003:327:25-37.

[21] Clinical and Laboratory Standard Institute (CLSI). Characterization and qualification of commutable reference material for laboratory medicine. Approved guideline. C53-A, vol. 30, no. 12; 2010.Wayne, Pennsylvania, USA.

[22] Yeo KT, Wu AH, Apple FS, et al. Multicenter evaluation of the Roche NT-proBNP assay and comparison to the Biosite Triage BNP assay. Clin Chim Acta 2003;338: 107-15.

[23] Sanz MP, Borque L, Rus A, Vicente B, Ramírez Y, Lasa L. Comparison of BNP and NT-proBNP assays in the approach to the emergency diagnosis of acute dyspnea. J Clin Lab Anal 2006;20:227-32.

[24] Bionda C, Bergerot C, Ardail D, Rodriguez-Lafrasse C, Rousson R. Plasma BNP and NT-proBNP assays by automated immunoanalyzers: analytical and clinical study. Ann Clin Lab Sci 2006;36:299-306.

[25] Koch AM, Rauh M, Zink S, Singer H. Decreasing ratio of plasma N-terminal pro-B-type natriuretic peptide and B-type natriuretic peptide according to age. Acta Paediatr 2006;95:805-9.

[26] Vittorini S, Prontera T, Zucchelli GC, Clerico A. Cardiac natriuretic hormones: methodological aspects. Immunol-Anal Biol Spec 2007;22:209-72.

[27] Clerico A, Prontera C, Emdin M, et al. Analytical performance and diagnostic accuracy of immunometric assays for the measurement of plasma BNP and NT-proBNP concentrations. Clin Chem 2005;51:445-7.

[28] Prontera C, Zucchelli CG, Vittorini S, Storti S, Emdin M, Clerico A. Comparison between analytical performances of polyclonal and monoclonal electrochemiluminescence immunoassays for NT-proBNP. Clin Chim Acta 2009;400:70-3.
[29] Prontera C, Storti S, Emdin M, et al. Comparison of a fully automated immunoassay with a point-of-care testing method for B-type natriuretic peptide. Clin Chem 2005;51:1274-6.

[30] Clerico A, Del Ry S, Giannessi D. Measurement of natriuretic cardiac hormones (ANP, BNP and related peptides) in clinical practice: the need for a new generation of immunoassay methods. Clin Chem 2000;46:1529-34.

[31] Storti S, Prontera C, Emdin M, et al. Analytical performance and clinical results of a fully automated MEIA system for BNP assay: comparison with a POCT method. Clin Chem Lab Med 2004;42:1178-85.

[32] Prontera C, Emdin M, Zucchelli GC, Ripoli A, Passino C, Clerico A. Analytical performance and diagnostic accuracy of a fully-automated electrochemiluminescent assay of the N-terminal fragment of brain natriuretic peptide in patients with cardiomyopathy: comparison with immunoradiometric assay methods for brain natriuretic peptide and atrial natriuretic peptide. Clin Chem Lab Med 2004;42: 37-44.

[33] Prontera C, Emdin M, Zucchelli GC, Ripoli A, Passino C, Clerico A. Automated electrochemiluminescent immunoassay for N-terminal pro-BNP compared with IRMAs for ANP and BNP in heart failure patients and healthy individuals (Letter). Clin Chem 2003;49:1552-4.

[34] Del Ry S, Giannessi D, Clerico A. Plasma brain natriuretic peptide measured by fully-automated immunoassay and by immunoradiometric assay compared. Clin Chem Lab Med 2001;39:446-50.

[35] Di Serio F, Ruggieri V, Varraso L, De Sario R, Mastrorilli A, Pansini N. Analytical evaluation of the Dade Behring Dimension RxL automated N-Terminal proBNP (NT-proBNP) method and comparison with the Roche Elecsys 2010. Clin Chem Lab Med 2005;43:1263-73.

[36] Vanderheyden M, Bartunek, Claeys G, Manoharan G, Beckers JF, Ide L. Head to head comparison of $\mathrm{N}$-terminal pro-B-type natriuretic peptide and B-type natriuretic peptide in patients with/without left ventricular systolic dysfunction. Clin Biochem 2006;39:640-5.

[37] Rawlins ML, Owen WE, Roberts WL. Performance characteristics of four automated natriuretic peptide assays. Am J Clin Pathol 2005;123:439-45.

[38] Emdin M, Passino C, Clerico A. Natriuretic peptide assays revisited: do we need pro-B-type natriuretic peptide? J Am Coll Cardiol 2011;57:1396-8.

[39] Clerico A, Vittorini S, Passino C. Measurement of the pro-hormone of brain type of natriuretic peptide (proBNP): methodological consideration and pathophysiological relevance. Clin Chem Lab Med 2011;49:1949-54.

[40] Giuliani I, Rieunier F, Larue C, et al. Assay for measurement of intact B-type natriuretic peptide prohormone in blood. Clin Chem 2006;52:1054-61.

[41] Macheret F, Boerrigter G, McKie P, et al. Pro-B-type natriuretic peptide 1-108 circulates in the general community: plasma determinants and detection of left ventricular systolic dysfunction. J Am Coll Cardiol 2011;57:1386-95. 